PROCEEDINGS OF THE

AMERICAN MATHEMATICAL SOCIETY

Volume 136, Number 12, December 2008, Pages 4413-4416

S 0002-9939(08)09411-2

Article electronically published on July 1, 2008

\title{
A TOPOLOGICAL REFLECTION PRINCIPLE EQUIVALENT TO SHELAH'S STRONG HYPOTHESIS
}

\author{
ASSAF RINOT
}

(Communicated by Julia Knight)

\begin{abstract}
We notice that Shelah's Strong Hypothesis is equivalent to the following reflection principle:

Suppose $\langle X, \tau\rangle$ is a first-countable space whose density is a regular cardinal, $\kappa$. If every separable subspace of $X$ is of cardinality at most $\kappa$, then the cardinality of $X$ is $\kappa$.
\end{abstract}

\section{INTRODUCTION}

Background. The Generalized Continuum Hypothesis (GCH) is equivalent to the assertion that $\lambda^{\mathrm{cf}(\lambda)}=\lambda^{+}$for every infinite cardinal $\lambda$. The Singular Cardinals Hypothesis $(\mathrm{SCH})$ is the assertion that $\lambda^{\mathrm{cf}(\lambda)}=\lambda^{+}$for every (obviously, singular) cardinal $\lambda$ satisfying $2^{\mathrm{cf}(\lambda)}<\lambda$.

Consider $[\lambda]^{\operatorname{cf}(\lambda)}$, the family of all subsets of $\lambda$ whose cardinality is $\operatorname{cf}(\lambda)$. It is not hard to see that $\lambda^{\operatorname{cf}(\lambda)}=2^{\operatorname{cf}(\lambda)} \cdot \operatorname{cof}\left([\lambda]^{\operatorname{cf}(\lambda)}\right)$, where $\operatorname{cof}\left([\lambda]^{\operatorname{cf}(\lambda)}\right)$ denotes the minimal cardinality of a cofinal subfamily $\mathcal{S}$; i.e., $\mathcal{S} \subseteq[\lambda]^{\operatorname{cf}(\lambda)}$, and for all $A \in[\lambda]^{\operatorname{cf}(\lambda)}$, there exists $B \in \mathcal{S}$ with $A \subseteq B$.

So, the idea motivating the definition of the $\mathrm{SCH}$ is to actually assert that $\operatorname{cof}\left([\lambda]^{\operatorname{cf}(\lambda)}\right)=\lambda^{+}$for every singular cardinal $\lambda$. However, if $\lambda$ is a singular cardinal and $2^{\operatorname{cf}(\lambda)}$ is quite large, then $\mathrm{SCH}$ gives us no information on the value of $\operatorname{cof}\left([\lambda]^{\operatorname{cf}(\lambda)}\right)$. To overcome this deficit, a list of alternative cardinal-arithmetic hypotheses has been introduced in $\S 6$ of $[8$. The first item of this list is referred in Shelah's paper [7] as The Strong Hypothesis (and we denote it by SSH).

To define the $\mathbf{S S H}$, fix a singular cardinal $\lambda$. Let us say that $\langle\lambda, \mathbf{a}, \mathcal{D}\rangle$ is an appropriate triplet iff $\mathbf{a} \subseteq \lambda$ is a set of $\operatorname{cf}(\lambda)$ many regular cardinals satisfying $\sup (\mathbf{a})=\lambda$, and $\mathcal{D}$ is an ultrafilter over a containing no bounded subsets. Consider the ultraproduct $\prod \mathbf{a} / \mathcal{D}$. It is a linearly ordered set and $\operatorname{cf}\left(\prod \mathbf{a} / \mathcal{D}\right) \geq \lambda^{+}$. Finally, define the pseudopower of $\lambda, \operatorname{pp}(\lambda)$, as the supremum of the following set:

$$
\operatorname{PP}(\lambda):=\left\{\operatorname{cf}\left(\prod \mathbf{a} / \mathcal{D}\right) \mid\langle\lambda, \mathbf{a}, \mathcal{D}\rangle \text { is an appropriate triplet }\right\} .
$$

Then $\lambda^{+} \leq \operatorname{pp}(\lambda) \leq \operatorname{cof}\left([\lambda]^{\operatorname{cf}(\lambda)}\right) \leq \lambda^{\operatorname{cf}(\lambda)}$. Thus, as the reader might expect, the SSH states that $\operatorname{pp}(\lambda)=\lambda^{+}$for every singular cardinal $\lambda$.

Received by the editors September 28, 2007, and, in revised form, November 3, 2007.

2000 Mathematics Subject Classification. Primary 03E04; Secondary 54G15, 03 E65.

Key words and phrases. Shelah's Strong Hypothesis, first countable, countably tight.

The author would like to thank his Ph.D. advisor, M. Gitik, for his comments and remarks.

(C)2008 American Mathematical Society 
Results. In this paper, we introduce a characterization of the SSH in purely topological terms. All arguments are standard. Perhaps the only challenge of this characterization lies in the need to formulate a topological principle that filters out the value of $2^{\mathrm{cf}(\lambda)}$. Our approach to establish this filtering is to formulate $\mathrm{SSH}$ as a reflection principle (for more on such principles, see [2]).

We mention that a topological characterization of the $\mathrm{SCH}$ was given in 3 . It is an interesting fact that while the non-trivial part of the proof of [3] was in showing that $\mathrm{SCH}$ implies their suggested topological principle, here the non-trivial part is in showing the other implication, i.e., that our topological principle implies the $\mathrm{SSH}$.

Notation. All ordinals in this paper are von Neumann ordinals. ICN denotes the class of infinite cardinal numbers, and REG denotes the class of regular infinite cardinals. The tightness of a topological space $\langle X, \tau\rangle$ is defined as $t(X)=\min \{\theta \in$ ICN $\left.\mid \forall A \subseteq X \forall x \in \bar{A} \exists B \in[A]^{\theta}(x \in \bar{B})\right\}$. The density of the space, $d(X)$, is the minimal (infinite) cardinality of a dense subset.

\section{RESUlts}

Theorem. The following are equivalent:

(1) Shelah's Strong Hypothesis.

(2) $\operatorname{cof}\left([\kappa]^{\theta}\right)=\kappa$ for all cardinals $\theta, \kappa$ satisfying $\theta<\operatorname{cf}(\kappa)$.

(3) Suppose $\langle X, \tau\rangle$ is a topological space and $t(X)<\operatorname{cf}(d(X))$. If every $B \in[X]^{t(x)}$ satisfies $|\bar{B}| \leq d(X)$, then $|X|=d(X)$.

(4) Suppose $\langle X, \tau\rangle$ is a first-countable space whose density is a regular cardinal, $\kappa$. If every separable subspace of $X$ is of cardinality at most $\kappa$, then the cardinality of $X$ is at most $\kappa$.

Proof. (1) $\Rightarrow(2)$ is well-known; see Theorem 6.3 of $[8$ or $\S 3$ of [5].

$(2) \Rightarrow(3)$ Let $\kappa=d(X)$ and $\theta=t(X)$. Pick a dense subset $D \in[X]^{\kappa}$. By $\theta<\operatorname{cf}(\kappa)$ and $(2)$, we have $\operatorname{cof}\left([\kappa]^{\theta}\right)=\kappa$, so let $\mathcal{S} \subseteq[D]^{\theta}$ be a cofinal subfamily of cardinality $\kappa$.

It follows from $t(X)=\theta$ and the definition of tightness that

$$
X=\bar{D}=\bigcup\left\{\bar{B} \mid B \in[D]^{\theta}\right\}=\bigcup\{\bar{B} \mid B \in \mathcal{S}\},
$$

and hence

$$
|X| \leq \sup \{|\bar{B}| \mid B \in \mathcal{S}\} \cdot|\mathcal{S}| \leq \kappa \cdot \kappa=\kappa .
$$

Since $|X| \geq d(X)=\kappa$, our proof is complete.

$(3) \Rightarrow(4)$ Suppose $\langle X, \tau\rangle$ has regular density and is first-countable. Clearly, $t(X)=\aleph_{0}$. Now, if $d(X)=\aleph_{0}$, then $X$ is separable and we are done. Otherwise, we have $t(X)<\operatorname{cf}(d(X))$, so appeal to (3).

$\neg(1) \Rightarrow \neg(4)$ Suppose the strong hypothesis fails. Then in particular, there exists a singular cardinal $\lambda$ of countable cofinality such that $\operatorname{pp}(\lambda)>\lambda^{+}$(see Claim 2.4 of $\left[9\right.$ ). $\operatorname{By} \operatorname{pp}(\lambda) \geq \lambda^{++}$and the convexity property of $\operatorname{PP}(\lambda)$ (see Conclusions 2.3 and 3.2 of [9]), we have $\lambda^{++} \in \operatorname{PP}(\lambda)$, so let us pick $\mathcal{D}$ and a such that $\langle\lambda, \mathbf{a}, \mathcal{D}\rangle$ is an appropriate triplet and $\operatorname{cf}\left(\prod \mathbf{a} / \mathcal{D}\right)=\lambda^{++}$.

Let $\left\langle f_{\alpha} \mid \alpha<\lambda^{++}\right\rangle$be a strictly increasing and cofinal sequence of the linearly ordered set $\prod \mathbf{a} / \mathcal{D}$. Since $|\mathbf{a}|=\aleph_{0}$, we may identify the two and assume that $\operatorname{dom}\left(f_{\alpha}\right)=\omega$ for all $\alpha<\lambda^{++}$. 
Note that if $I \subseteq \lambda^{++}$is of cardinality $\lambda^{++}$, then $\left\langle f_{\alpha} \mid \alpha \in I\right\rangle$ is cofinal as well. In particular, for such $I$, by a $\subseteq$ REG, there must exist some $n<\omega$ such that $\left\{f_{\alpha}(n) \mid \alpha \in I\right\}$ is uncountable.

Fix an injection $\psi:{ }^{<\omega} \lambda \rightarrow \lambda$ and let $y_{\alpha}:=\left\{\psi\left(f_{\alpha} \uparrow n\right) \mid 0<n<\omega\right\}$ for all $\alpha<\lambda^{++}$. Put $\kappa:=\lambda^{+}$and $\mathcal{F}:=\left\{y_{\alpha} \mid \alpha<\kappa^{+}\right\}$. Clearly, $\kappa \cap \mathcal{F}=\aleph_{1} \cap \mathcal{F}$, and hence we may assume that $\kappa \cap \mathcal{F}=\emptyset$.

We now define a topological space $\langle X, \tau\rangle$ similar to the one introduced by Mrówka in 4 .

Put $X=\kappa \uplus \mathcal{F}$. Each point of $\kappa$ will be isolated. For each $y \in \mathcal{F}$, assign the local base $\mathcal{B}_{y}:=\{\{y\} \cup(y \backslash a) \mid a \subseteq y$ is finite $\}$. Then $\langle X, \tau\rangle$ is indeed first-countable.

Notice that for any $A \subseteq \kappa$ and $B \subseteq \mathcal{F}$, we have

$$
\bar{A}=A \cup\{y \in \mathcal{F} \mid A \cap y \text { is infinite }\}, \quad \bar{B}=B .
$$

It follows that $\mathcal{F} \subseteq \bar{\lambda}$ and $X \subseteq \bar{\kappa}$. In particular, $d(X) \leq \kappa$. On the other hand, each point of $\kappa$ is isolated in $X$, and hence $d(X)=\kappa$.

Clearly, $|X|=\kappa^{+}$, so we are left with showing that any separable subspace of $X$ is of cardinality at most $\kappa$. Suppose toward a contradiction that $S \subseteq X$ is countable and there exist some $I \subseteq \kappa^{+}$of cardinality $\kappa^{+}$such that $S \cap y_{\alpha}$ is infinite for each $\alpha \in I$. By the remark above, fix $n^{*}<\omega$ and an uncountable $J \subseteq I$ such that $f_{\alpha}\left(n^{*}\right) \neq f_{\beta}\left(n^{*}\right)$ for all distinct $\alpha, \beta \in J$. It follows that

$$
\left\{( f _ { \alpha } \lceil m ) | n ^ { * } < m < \omega \} \cap \left\{\left(f_{\beta}\lceil m) \mid n^{*}<m<\omega\right\}=\emptyset,\right.\right.
$$

for all distinct $\alpha, \beta \in J$. Thus, letting $z_{\alpha}:=\left\{\psi\left(f_{\alpha} \uparrow m\right) \mid m \leq n^{*}\right\}$ for each $\alpha \in J$, we get that $\left\{S \cap\left(y_{\alpha} \backslash z_{\alpha}\right) \mid \alpha \in J\right\}$ is an uncountable family of mutually disjoint infinite subsets of $S$, a contradiction to $|S| \leq \aleph_{0}$.

\section{Closing Remarks}

(1) The preceding construction can be improved to make all separable subspaces countable, given that the $\mathrm{SSH}$ fails in a stronger sense.

More precisely, assuming $\operatorname{pp}(\lambda)>\lambda^{++}$for a cardinal $\lambda>\operatorname{cf}(\lambda)=\aleph_{0}$, there exists a 0-dimensional, locally compact, locally countable, first-countable, Hausdorff space, $\langle X, \tau\rangle$, with $c(X)=d(X)=\lambda,|X|=e(X)=w(X)=\lambda^{++}$, and, in addition, $|\bar{S}|=|S|$ for all $S \in[X]^{<\lambda}$. (For missing definitions, see [1].)

Note that $Y:=D\left(\lambda^{+}\right) \times X$, the topological product of a discrete space of cardinality $\lambda^{+}$with the above $\langle X, \tau\rangle$, will share the same properties of $\langle X, \tau\rangle$, except that it has $c(Y)=d(Y)=\operatorname{cf}(d(Y))=\lambda^{+}$.

(2) A sharp weakening of the SSH, the Prevalent Singular Cardinals Hypothesis (PSH), was introduced in [6] and shown to imply that any topological space of density and weight $\aleph_{\omega_{1}}$ is not hereditarily Lindelöf. It is an open problem whether this kind of topological property characterizes the PSH.

(3) It is our hope that this paper will contribute to popularizing the usage of weak cardinal arithmetic hypotheses (and their negations) in general topology.

\section{REFERENCES}

[1] R. E. Hodel. Cardinal functions. I. In Handbook of set-theoretic topology, pages 1-61. NorthHolland, Amsterdam, 1984. MR776620 (86j:54007)

[2] R. E. Hodel and J. E. Vaughan. Reflection theorems for cardinal functions. Topology Appl., 100(1):47-66, 2000. Special issue in honor of Howard H. Wicke. MR1731704 (2001b:54004) 
[3] M. Ismail and A. Szymanski. A topological equivalence of the singular cardinals hypothesis. Proc. Amer. Math. Soc., 123(3):971-973, 1995. MR1285997 (95k:03076)

[4] S. Mrówka. On completely regular spaces. Fund. Math., 41:105-106, 1954. MR0063650 $(16: 157 \mathrm{~b})$

[5] A. Rinot. On the consistency strength of the Milner-Sauer conjecture. Ann. Pure Appl. Logic, 140(1-3):110-119, 2006. MR2224053 (2007a:03067)

[6] A. Rinot. On topological spaces of singular density and minimal weight. Topology Appl., 155(3):135-140, 2007. MR2370368

[7] S. Shelah. Cardinal arithmetic for skeptics. Bull. Amer. Math. Soc. (N.S.), 26(2):197-210, 1992. MR 1112424 (92h:03071)

[8] S. Shelah. Advances in cardinal arithmetic. In Finite and infinite combinatorics in sets and logic (Banff, AB, 1991), volume 411 of NATO Adv. Sci. Inst. Ser. C Math. Phys. Sci., pages 355-383. Kluwer Acad. Publ., Dordrecht, 1993. MR1261217 (95h:03112)

[9] S. Shelah. $\aleph_{\omega+1}$ has a Jonsson algebra. In Cardinal Arithmetic, pages 34-116. Oxford Logic Guides 29, Oxford Univ. Press, 1994. MR1318912(96e:03001)

School of Mathematical Sciences, Tel Aviv University, Tel Aviv 69978, Israel

E-mail address: assaf@rinot.com

URL: http://www.tau.ac.il/ rinot 